\title{
Leaky LMS Algorithm and Fractional Brownian Motion Model for GNSS Receiver Position Estimation
}

\author{
Jean-Philippe Montillet \\ Environmental Geodesy Earth Physics \\ Research School of Earth Sciences \\ The Australian National University \\ Canberra, Australia \\ jeanfi_montillet@yahoo.fr
}

\author{
Kegen $\mathrm{Yu}$ \\ Satellite Navigation and Positioning Laboratory \\ School of Surveying and Spatial Information Systems \\ University of New South Wales \\ Sydney, Australia \\ kegen.yu@ieee.org
}

\begin{abstract}
This paper presents a new approach for smoothing long time series of position estimates of ground GNSS (global navigation satellite system) receivers. The fractional Brownian motion ( $\mathrm{fBm})$ model is employed to describe the position coordinate estimates that have long-range dependencies. A new and low-complexity method is proposed to estimate the Hurst parameter and the simulation results show that the new method achieves good accuracy and low complexity. A modified leaky least mean squares (ML-LMS) estimator is proposed to filter the long time series of the position coordinate estimates, which uses the Hurst parameter estimates to update the filter tap weights. Simulation results demonstrate that this ML-LMS estimator outperforms the classic LMS estimator considerably in terms of both accuracy and convergence.
\end{abstract}

Keywords-GNSS positioning; fractional Browinian motion model; Hurst parameter estimation; modified leaky LMS estimator

\section{INTRODUCTION}

The Brownian motion is a well-known model originally used to describe the movement of a particle in a liquid [1]. Due to its stochastic properties, the model has been used in many research fields such as in finance and engineering [2, 3]. The fractional Brownian motion $(\mathrm{fBm})$ based on the Hurst $(\mathrm{H})$ parameter was developed to model long-run non-periodic statistical dependences of time series [4]. This $\mathrm{fBm}$ model has been successfully applied in chemistry with the time series of the turbulences of the pressure signal of an airlift reactor [5] and in telecommunications for the study of specific networks [3]. In reality long time series of many processes cannot simply be modeled either as Brownian motion or as Gaussian. Thus, the $\mathrm{fBm}$ model gives some degree of freedom to characterize the process noise or measurement errors of the time series thanks to the $\mathrm{H}$ parameter which was introduced in [6] for studying the long-run non-periodic statistical dependences of time series in hydrology. This parameter is defined in the interval $[0,1]$. If $\mathrm{H}<0.5$ the increments of the process are negatively correlated and the statistics of the process noise are more Gaussian, whereas if $\mathrm{H}>0.5$ the increments are positively correlated and the statistics of the process noise are more Brownian. The $\mathrm{fBm}$ becomes the standard Brownian motion when $\mathrm{H}=0.5$. Note that the $\mathrm{fBm}$ process exhibits long-range dependence when $\mathrm{H}>0.5$.
This paper investigates methods for improving the positional accuracy of ground receivers which are based on the global navigation satellite system (GNSS). The $\mathrm{fBm}$ model is utilized to model the long time series of position coordinate estimates of the GNSS receiver. For simplicity, only one-dimensional coordinate data are employed in this work for the development of the theory. The degree of fitting of the $\mathrm{fBm}$ model to the time series of the receiver coordinate estimates will be studied through choosing the appropriate values of the $\mathrm{H}$ parameter. Knowledge about the $\mathrm{H}$ parameter is important and a number of different methods can be used to estimate this parameter. In this paper, an alternative technique for estimating the $\mathrm{H}$ parameter is proposed, by windowing the data set and using the self-similarity property of the time series. Although it is similar to the aggregated variance method developed in [7], we reformulate the solution in block matrices. In addition, we show that the proposed $\mathrm{H}$-parameter algorithm reaches the minimum variance unbiased (MVU) estimator when using the Gauss-Markov model to describe the fluctuations of the $\mathrm{H}$ parameter. The MVU estimator was previously underlined in the application of the $\mathrm{fBm}$ model to network security management [8].

The LMS algorithm has found wide application in many areas of adaptive signal processing and control. It is a fact that the classic LMS filter is optimum when the measurement noise/error is Gaussian as explained in [9]. Recently the leakyLMS (L-LMS) algorithms have been proposed to improve the performance of the LMS algorithm especially regarding the stability and convergence [10]. In this paper, we exploit the LLMS algorithm to smooth the GNSS position coordinate estimates. In particular, the $\mathrm{fBm}$ model and the $\mathrm{H}$ parameter estimation are combined with the L-LMS algorithm to form a modified L-LMS (ML-LMS). The use of the $\mathrm{fBm}$ model to adaptive filtering is relatively new since there is only one relevant reference in the literature [11], where a modified variable step-size LMS is proposed for adaptive channel equalization. Note that another approach for GNSS positional data smoothing is the Kalman filter [12], which is not considered in this work, but it is worth further investigation in further work. The remainder of the paper is organized as 
follows. The following section describes the $\mathrm{fBm}$ model and the H parameter estimation. Section III develops the proposed ML-LMS algorithm. Section IV shows simulation results based on field measurements, and Section V concludes the paper.

\section{Fractional BROWNIAN MOTION MODEL AND HURST PARAMETER ESTIMATION}

\section{A. Fractional Brownian Motion Model}

As mentioned previously, the fractional Brownian motion has been studied for more than a number of decades. The $\mathrm{fBm}$ model is defined as:

$$
\begin{array}{r}
B_{H}(t, \omega)-B_{H}(0, \omega)=\frac{1}{\Gamma(H+1 / 2)}\left\{\int _ { - \infty } ^ { 0 } \left[(t-s)^{H-1 / 2}-\right.\right. \\
\left.(-s)^{H-1 / 2}\right] d B(s, \omega)+\int_{0}^{t}\left[(t-s)^{\mathrm{H}-1 / 2} d B(s, \omega)\right\}
\end{array}
$$

where $t$ denotes time, $\omega$ denotes the set of all the values of a random function, $H$ is the $H$ parameter, $B_{H}(0, \omega)$ is the starting value and $B(t, \omega)$ is the ordinary Brownian motion. Note that $t$ has to be positive in the general expression of the $\mathrm{fBm}$. A nice overview of empirical studies to calculate the $\mathrm{H}$ parameter can be seen in [7]. Early works were based on the regression of the time series and the property of self-similarities was employed to compute the $\mathrm{H}$ parameter (e.g. [6], [13]), but this estimation method has a high standard deviation. By windowing the time series, [7] and [14] developed the auto-regressive moving average (ARMA) and fractional auto-regressive integrated moving average (FARIMA) methods to compute the H. All these methods mentioned above are generally referred to as aggregated variance method. More recently wavelet theory has been applied to this engineering problem such as in [15].

\section{B. Estimation of the Hurst Parameter}

First, let us recall the definition of self-similarities between two functions from [4].

Definition: The increments of a random function $\{\mathrm{X}(t, \infty)$; $+\infty>t>-\infty\}$ will be said to be self-similar with parameter $H$ $(H \geq 0)$ if for any $h>0$ and any $t_{0}$,

$$
\begin{gathered}
\left\{\mathrm{X}\left(t_{0}+h \tau, \omega\right)-\mathrm{X}\left(t_{0}, \omega\right)\right\} \triangleq\left\{h ^ { - H } \left[\mathrm{X}\left(t_{0}+\tau, \omega\right)\right.\right. \\
\left.\left.-\mathrm{X}\left(t_{0}, \omega\right)\right]\right\}
\end{gathered}
$$

This definition relates directly to the $\mathrm{fBm}$ and the increments $B_{H}(t, \omega)$ are stationary and self-similar with parameter $H$. As mentioned earlier, the long-run non-periodic statistical dependences of the time series are related to the $H$ parameter. Thus, the approach taken in this work to model the single point positioning times series of the coordinates is directly based on (2) and the self-similarity of the $\mathrm{fBm}$. Thus, the $H$ parameter can be extracted from (2), producing

$$
H(h, \tau) \triangleq \frac{-1}{\log (h)} \log \left(\frac{|\mathrm{X}(t+\tau)-\mathrm{X}(t)|}{|\mathrm{X}(t+h \tau)-\mathrm{X}(t)|}\right)
$$

where $\mathrm{X}(t)$ is the measurement data at time $t, \tau$ is an arbitrary value, $h$ is a variable which varies during the data fitting process. Clearly, $H$ is a function of the parameters $h$ and $\tau$ which are selected by the user. In the event that for a given $\tau$ the variable $h$ takes different values so that it can be expressed in vector form as:

$$
\boldsymbol{h}=\left[h_{1}, h_{2}, h_{3}, \ldots, h_{L}\right]^{T}, \quad h_{i}<h_{i+1}
$$

Then, (3) can be rewritten in matrix form as:

$$
\mathbf{H}=\mathbf{D}(\alpha(\mathrm{h})) \times \mathbf{U}(\mathrm{h}) \times \mathbf{1}
$$

where $\mathbf{1}$ is the column vector of all ones with length $M$ and

$$
\begin{aligned}
& \mathbf{H}=\left[\begin{array}{llll}
H_{1} & H_{2} & \cdots & H_{L}
\end{array}\right]^{T} \\
& \mathbf{D}(\alpha(h))=\operatorname{diag}\left\{\alpha\left(h_{1}\right), \quad \alpha\left(h_{2}\right), \quad \cdots, \quad \alpha\left(h_{L}\right)\right\} \\
& \alpha\left(h_{i}\right)=\left(\log \left(h_{i}\right)\right)^{-1} \\
& \mathbf{U}(h)=\left(\begin{array}{cccc}
u\left(h_{1}, 1\right) & u\left(h_{1}, 2\right) & \cdots & u\left(h_{1}, M\right) \\
u\left(h_{2}, 1\right) & u\left(h_{2}, 2\right) & \cdots & u\left(h_{2}, M\right) \\
\vdots & \vdots & \ddots & \vdots \\
u\left(h_{L}, 1\right) & u\left(h_{L}, 2\right) & \cdots & u\left(h_{L}, M\right)
\end{array}\right) \\
& u\left(h_{i}, 1\right)=\log \frac{|\mathrm{X}(1+\tau)-\mathrm{X}(1)|}{\left|\mathrm{X}\left(1+h_{i} \tau\right)-\mathrm{X}(1)\right|}
\end{aligned}
$$

where the initial time instant $t$ is set to one for simplicity. Moreover, the definition of $u\left(h_{i}, 1\right)$ requires that $\tau$ is defined in $\mathbb{Z}^{+}$whereas $h$ in $\mathbb{Z}$. $\mathbf{U}$ then contains the time series of $\mathrm{X}$ with different window lengths, and (3) may be seen as an autoregressive-moving-average (ARMA) model (see [9]). Thus, the $\mathrm{H}$ parameter can be estimated by:

$$
\hat{H}=\frac{1}{L} \sum_{i=1}^{L} H_{i}
$$

Property 1: If $\mathbf{H} \sim \mathcal{N}\left(\mathbf{D}(\alpha(h)) \times \mathbf{U}(h) \times \mathbf{1}, \sigma^{\mathbf{2}} \mathbf{I}\right)$, then $\widehat{H}$ given by (7) is the minimum variance unbiased estimate of the Hurst parameter with a variance equal to $\frac{\sigma^{2}}{L}$.

Proof: We assume the Gauss-Markov model of the Hurst parameter $H$. Then, (4) becomes

$$
\mathbf{H}=\mathbf{D}(\alpha(h)) \times \mathbf{U}(h) \times \mathbf{1}+\mathbf{W}
$$

where $\mathbf{W}$ is the error vector due to the approximation of the Hurst parameter and it is assumed to have a Gaussian distribution with a zero mean and a covariance matrix equal to $\sigma^{2} \mathbf{I}$. From the parameter estimation theory [16], the performance of the minimum variance unbiased (MVU) estimator with this linear model approaches the Cramer-Rao Lower Bound. Thus, the MVU estimate of the Hurst parameter is given by (7) with a variance equal to $\frac{\sigma^{2}}{L}$.

\section{MODIFIED LEAKY LMS ALGORITHM FOR SMOOTHING POSITION ESTIMATES}

\section{A. Statistical models for the adaptive filtering}

In this paper the time series of interest is the coordinates of a ground receiver (in WGS 84) triangulated with GPS pseudoranges in single point positioning. For a fixed receiver, it is interesting to model the long-term and short-term correlation structure of these time series data [14] when recording the rover's position during hours or days of observation. The choice of this model is to give some degree 
of freedom instead of using the classic Gaussian model which is generally used in satellite-based positioning theory [17].

One way to constrain the variance of the receiver position estimates is generally to use a Kalman filter in backward filtering. Here, we intend to develop a simpler approach by using a LMS filter as the case study is limited to a static receiver. The research community has already investigated the application of the adaptive filters to constrain the error of time-of-arrival measurements in sensor localization [18]. The authors in [19] developed a LMS filter to reduce the recurrent multipath error due to satellite geometry on the double difference observations for a static rover. As a result, the double difference residuals have smaller variances and the rover's coordinate estimates are steadier.

The LMS filter is an iterative algorithm which adapts a stationary random process $(\mathrm{v}(n))$ to a desired output $(\mathrm{d}(n))$ with the same stochastic properties. Let us define the discretetime processes:

$$
\mathbf{v}_{k}=\mathbf{x}_{k}-x_{0}, \quad \mathbf{d}_{k}=\mathbf{g}_{k}-x_{0}
$$

where the time series of the receiver's coordinate estimates is one dimensional, given by $\mathbf{x}_{k}=[x(k), x(k+1), \ldots, x(k+$ $N)]^{T} . \mathbf{x}_{k}, \mathbf{v}_{k}, \mathbf{g}_{k}$ and $\mathbf{d}_{k}$ are all in $\mathbb{R}^{N}$. As $\mathbf{v}_{k}$ is the time series that we want to adapt to a desire time series $\mathbf{d}_{k}$, it follows that $\sigma_{d}^{2}<\sigma_{v}^{2}$. In other words, it can be seen that $\mathbf{d}_{k}$ is the time series coordinate of the same rover, but filtered (e.g. moving average) or coming from another sensor (e.g. Inertial Measurement Unit - see [20]) with smaller variances. $x_{0}$ is the true rover's coordinate. Due to multipath propagation, $\mathbf{v}_{k}$ and $\mathbf{d}_{k}$ are not necessarily zero-mean Gaussian distributed, but follow a fractional Brownian motion model as:

$$
\mathbf{v}_{k} \sim f B m\left(H_{v}, \sigma_{v}^{2}, \mu_{v}\right), \quad \mathbf{d}_{k} \sim f B m\left(H_{d}, \sigma_{d}^{2}, \mu_{d}\right)
$$

where $H_{v}$ and $H_{d}$ are the Hurst parameters satisfying | $H_{1}-$ $H_{2} \mid<\xi_{1}$ where $\xi_{1}$ is a small positive number. To guarantee the convergence of the LMS filter and unbiased solution, it is important to have a strong hypothesis on the means: I $\mu_{v}-$ $\mu_{d} \mid<\xi_{2}$ where $\xi_{2}$ is a very small number.

In the LMS algorithm described in [9] the filter output is given by $y_{k}=\mathbf{w}_{\mathbf{k}}^{\mathbf{T}} \mathbf{v}_{k}$ where $\mathbf{w}_{k}$ is the filter tap weight vector. The error signal is defined as $e_{k}=d_{k}-y_{k}$ so that the mean square error is given by:

$$
E\left[e_{k}^{2}\right]=E\left[d_{k}^{2}\right]-2 \mathbf{p}^{T} \mathbf{w}_{k}+\mathbf{w}_{k}^{T} \mathbf{R} \mathbf{w}_{k}
$$

where $\mathrm{E}[$.$] is the expectation operator, \mathbf{p} \triangleq E\left[d_{k} \mathbf{v}_{k}\right]$ and $\mathbf{R} \triangleq E\left[\mathbf{v}_{k}^{\mathrm{T}} \mathbf{v}_{k}\right]$. The LMS filter is an iterative algorithm which updates the tap weight vector $\mathbf{w}_{k}$ according to:

$$
\mathbf{w}_{k+1}=\mathbf{w}_{k}+2 \mu e_{k} \mathbf{v}_{k}
$$

where $\mu$ is a selectable parameter. It has been shown that in the case of uncorrelated Gaussian data, $\boldsymbol{w}_{k}$ converges to the Wiener solution with $0<\mu<1 /(3 \operatorname{tr}(\mathbf{R}))$ (see [10]). Each of the weight vector elements exponentially relaxes to its optimal value with a time constant inversely proportional to the $n$-th eigenvalue of $\mathbf{R}$. Further, the eigenvalue spread, defined as the ratio of the largest eigenvalue over the smallest one $\left(\lambda_{\max } / \lambda_{\min }\right)$, plays a critical role in the convergence of the LMS filter.

\section{B. Modified Leaky LMS Algorithm}

Property 2: Let us consider two $\mathrm{fBm}$ processes with Hurst parameters $H_{a}$ and $H_{b}$ constrained by $H_{a}<0.5$ and $H_{b}>0.5$, and with covariance matrix $\mathbf{R}_{a}$ and $\mathbf{R}_{b}$, respectively. Then, the eigenvalue spreads of the two covariance matrices satisfy:

$$
\frac{\lambda_{\max }^{(a)}}{\lambda_{\min }^{(a)}}<\frac{\lambda_{\max }^{(b)}}{\lambda_{\min }^{(b)}}
$$

where the superscripts are used to denote the corresponding covariance matrices.

Proof : as shown in [11], the $N \times N$ auto-covariance matrix $\mathbf{R}(n)$ of a discrete $\mathrm{fBm}$ can be decomposed to be $\mathbf{R}(n)=$ $\mathbf{Q}(n) \boldsymbol{\Lambda}(n) \mathbf{Q}(n)^{T}$. Then, the auto-covariance matrix can be approximated as $\widehat{\mathbf{R}}(n)=\mathbf{Q} \widehat{\boldsymbol{\Lambda}}(n) \mathbf{Q}^{T}$ for large $n$ where $\mathbf{Q}$ is a constant orthogonal matrix and $\widehat{\Lambda}(n)=\operatorname{diag}\left\{\lambda_{1}, \lambda_{2}, \ldots, \lambda_{N}(n)\right\}$. Thus only the largest eigenvalue is time dependent for large $n$ and given by:

$$
\begin{aligned}
& \lambda_{N}(n, H)=\frac{1}{\Gamma(2 H+1)|\sin H|}\left\{2 N n^{2 H}+\right. \\
& \left.2 H n^{2 H-1}(N-1) N-\frac{2}{N} \sum_{i=1}^{N-1} i(N-i)^{2 H}\right\}
\end{aligned}
$$

The eigenvalue spread is then given by

$$
\begin{aligned}
& \frac{\lambda_{\max }}{\lambda_{\min }}=\frac{\lambda_{N}(n, H)}{\lambda_{1}(H)} \\
& =\frac{2 N n^{2 H}+2 H n^{2 H-1}(N-1) N-\frac{2}{N} \sum_{i=1}^{N-1} i(N-i)^{2 H}}{\lambda_{1}(H) \Gamma(2 H+1)|\sin H|}
\end{aligned}
$$

It can be shown that the denominator in (15) basically varies between 0.12 and 0.148 when the $\mathrm{H}$ parameter ranges from 0.1 to 0.9. Also, the value of the denominator when $H<0.5$ is greater than that when $H>0.5$ in general. On the other hand, if $N \gg 1$ and $n$ is large such as $n>N$, the value of the numerator increases quickly with respect to the $\mathrm{H}$ parameter. Therefore, the eigenvalue spread when $H<0.5$ is smaller than that when $H>0.5$

When the eigenvalue spread increases, the rate of convergence of the LMS algorithm decreases [10]. In our case study, the eigenvalue spread increases significantly as $H$ is close to 1 and thus it is necessary to improve the convergence rate. Similarly to the cost function defined for the variable leaky LMS algorithm in [10], our cost function is defined as

$$
J_{k}=e_{k}^{2}+\gamma H \mathbf{w}_{k}^{T} \mathbf{w}_{k}
$$

where clearly the $\mathrm{H}$ parameter is directly used to adjust the cost function. Then, the tap weight vector is updated by:

$$
\mathbf{w}_{k+1}=(1-2 \mu \gamma H) \mathbf{w}_{k}+2 \mu e_{k} \mathbf{v}_{k}
$$


Clearly, the cost function in (16) is devised to achieve a good trade-off between the optimal LMS estimator when the noise is Gaussian $(\mathrm{H}<0.5)$ and good convergence when the noise is $\mathrm{fBm}(\mathrm{H}>0.5)$. Note that no real data are used through this study and a $\mathrm{fBm}$ noise function generates the receiver time series.

\section{Simulation RESUlts}

In this section, the novel approach to calculate the $\mathrm{H}$ parameter is tested against other ones previously published in the scientific literature. Also, the ML-LMS algorithm developed in Section III is evaluated through simulations using field measurements.

\section{A. Test of the novel method to calculate the H parameter}

The $\mathrm{fBm}$ time series are generated using the Matlab library function wfbm (see Matlab 7.2) with the true $\mathrm{H}$ parameter as input (first column of Table 1). Then, the $\mathrm{H}$ parameter is estimated using our proposed method developed in Section II$B$ and the results are denoted by subscript "p". For performance comparison, the results using two existing methods, namely the wavelet method (wav) and the auto regressive method (RS), are also listed. Note that the estimation of the Hurst parameter with the wavelet and RS methods is performed using the Matlab function wfbmesti.

The results are averaged on 1000 simulations and the mean and standard deviation are extracted for each case. The results in Table 1 clearly show that the proposed method slightly outperforms the wavelet method, whereas the performance of the RS method is the worst.

Table 1. Accuracy of the $\mathrm{H}$ parameter estimation.

\begin{tabular}{|c|c|c|c|c|c|c|}
\hline \multirow{2}{*}{$\mathrm{H}$} & \multicolumn{6}{|c|}{ Statistics } \\
\cline { 2 - 7 } & $\boldsymbol{\mu}_{\boldsymbol{p}}$ & $\boldsymbol{\mu}_{\text {wav }}$ & $\boldsymbol{\mu}_{\boldsymbol{R}}$ S & $\boldsymbol{s t d}_{\boldsymbol{p}}$ & $\boldsymbol{s t d}_{\text {wav }}$ & $\boldsymbol{s t d}_{\boldsymbol{R} S}$ \\
\hline 0.1 & 0.162 & 0.171 & 0.037 & 0.042 & 0.041 & 0.069 \\
\hline 0.2 & 0.233 & 0.238 & 0.086 & 0.042 & 0.042 & 0.066 \\
\hline 0.3 & 0.317 & 0.323 & 0.206 & 0.041 & 0.044 & 0.069 \\
\hline 0.43 & 0.432 & 0.434 & 0.355 & 0.04 & 0.044 & 0.070 \\
\hline 0.52 & 0.518 & 0.517 & 0.463 & 0.039 & 0.045 & 0.068 \\
\hline 0.6 & 0.597 & 0.596 & 0.549 & 0.038 & 0.044 & 0.072 \\
\hline 0.75 & 0.746 & 0.741 & 0.709 & 0.041 & 0.048 & 0.073 \\
\hline 0.81 & 0.804 & 0.804 & 0.771 & 0.042 & 0.049 & 0.076 \\
\hline 0.95 & 0.918 & 0.944 & 0.888 & 0.041 & 0.049 & 0.069 \\
\hline
\end{tabular}

\section{B. Performances of the modified Leaky-LMSin static scenario}

According to the model in (10), a zero-mean Gaussian noise with standard deviation equal to $\sigma_{n}=25 \mathrm{~m}$ is added to the input signal $\left(\mathbf{v}_{k}\right)$ so that it has a higher standard deviation than the reference signal $\left(\mathbf{d}_{k}\right)$. The $\mathrm{H}$ parameters of the two time series are set to be the same and $\mu_{v}$ and $\mu_{d}$ are equal to zero. The $\mathrm{fBm}$ processes are generated using the Matlab library function in the same way as in Section IV-A. Thus, the variances $\sigma_{v}^{2}$ and $\sigma_{d}^{2}$ are not a parameter chosen by the user. The simulation testbed uses various $H$ parameter values and for each value the error mean and standard deviation are averaged over 1000 simulation runs. The proposed leaky LMS algorithm is compared against the standard LMS method and the original Leaky LMS algorithm (L-LMS) as shown in Tables 2 and 3 . The results show that all the adaptive algorithms perform well using this testbed. The proposed leaky LMS (ML-LMS) and the LMS algorithm produce similar performance for $H<0.5$ (in Gaussian noise), whereas if $H$ is close to 1 the performance of the proposed algorithm is similar to that of the L-LMS. Thus the simulations confirm the mathematics developed in the previous sections.

The convergence rate is evaluated through observing the parameter $\mu$ defined in the cost functions of the adaptive algorithms. It is equal to $0.1(\operatorname{tr}(\boldsymbol{\Lambda}))^{-1}$ where $\boldsymbol{\Lambda}$ is the diagonal matrix resulting from the decomposition of the covariance matrix of the measurements (R). Fig. 1 and Fig. 2 show the curves of the estimation errors with the two adaptive algorithms (LMS and ML-LMS). The L-LMS is not shown here as the performances are close to the ML-LMS as seen in the previous Tables. The statistics of the adaptive filtering error is improved when using the ML-LMS. The figures confirm that the inclusion of the $\mathrm{fBm}$ model in the adaptive filter helps to smooth the first and second moment order statistics of a time series with a variable $\mathrm{H}$ parameter. Similar results were also observed in [10].

\section{CONCLUSIONS}

A new method was proposed to estimate the $\mathrm{H}$ parameter of the $\mathrm{fBm}$ model that is used to describe the long time series of the position coordinate estimates of GNSS receivers. The method is directly derived from the self-similarity property of the $\mathrm{fBm}$. The results demonstrated that this method significantly outperforms the RS method in terms of the accuracy of the $\mathrm{H}$ parameter estimation. It also slightly outperforms the wavelet method on average. Also, the adaptive filter was employed for GNSS positioning and a MLLMS algorithm was developed to filter the long time series of the estimates of GNSS receiver coordinates. The coast function of the Leaky LMS is modified with the introduction of the $\mathrm{H}$ parameter, resulting in the ML-LMS algorithm. The results demonstrated that the proposed method outperforms the LMS algorithm in terms of both accuracy and convergence rate. Future work should be focused on the comparison with the Kalman filter performances and on tracking moving objects. In addition, ongoing work intends to develop better techniques for the ML-LMS algorithm to achieve better performance.

Table 2. Mean and standard deviation of the three algorithms using a simulated reference series.

\begin{tabular}{|c|c|c|c|c|}
\hline \multirow{2}{*}{} & \multicolumn{2}{|c|}{$\begin{array}{c}\text { Time Series A } \\
0<\mathrm{H}<0.5\end{array}$} & \multicolumn{2}{c|}{$\begin{array}{c}\text { Time Series B } \\
0.5<\mathrm{H}<1\end{array}$} \\
\cline { 2 - 5 } & Mean $(\mathrm{m})$ & $\mathrm{STD}(\mathrm{m})$ & Mean $(\mathrm{m})$ & STD $(\mathrm{m})$ \\
\hline Original & -0.01 & 0.352 & -6.157 & 9.189 \\
\hline Ref & -0.01 & 0.343 & -6.157 & 9.180 \\
\hline LMS & 0.085 & 0.055 & 1.500 & 1.434 \\
\hline
\end{tabular}




\begin{tabular}{|c|c|c|c|c|}
\hline L-LMS & 0.084 & 0.054 & 1.199 & 1.150 \\
\hline ML-LMS & 0.082 & 0.053 & 1.199 & 1.150 \\
\hline
\end{tabular}

[3] I. Norros, "On the use of fractional Brownian motion in the theory of connectionless networks," IEEE Journal on Selected Areas of Communications, vol. 13, no. 6, pp. 953-962, Aug. 1995.

Table 3. Mean and standard deviation of the three algorithms using a median filter.

\begin{tabular}{|c|c|c|c|c|}
\hline & \multicolumn{2}{|c|}{$\begin{array}{c}\text { Time Series A } \\
0<\mathrm{H}<0.5\end{array}$} & \multicolumn{2}{c|}{$\begin{array}{c}\text { Time Series B } \\
0.5<\mathrm{H}<1\end{array}$} \\
\hline & Mean $(\mathrm{m})$ & STD $(\mathrm{m})$ & Mean $(\mathrm{m})$ & STD $(\mathrm{m})$ \\
\hline Original & 0.118 & 0.373 & -6.157 & 9.189 \\
\hline Med & 0.118 & 0.365 & -6.267 & 9.074 \\
\hline LMS & 0.088 & 0.058 & 1.892 & 1.701 \\
\hline L-LMS & 0.074 & 0.051 & 1.398 & 1.301 \\
\hline $\begin{array}{c}\text { ML- } \\
\text { LMS }\end{array}$ & 0.074 & 0.051 & 1.398 & 1.301 \\
\hline
\end{tabular}

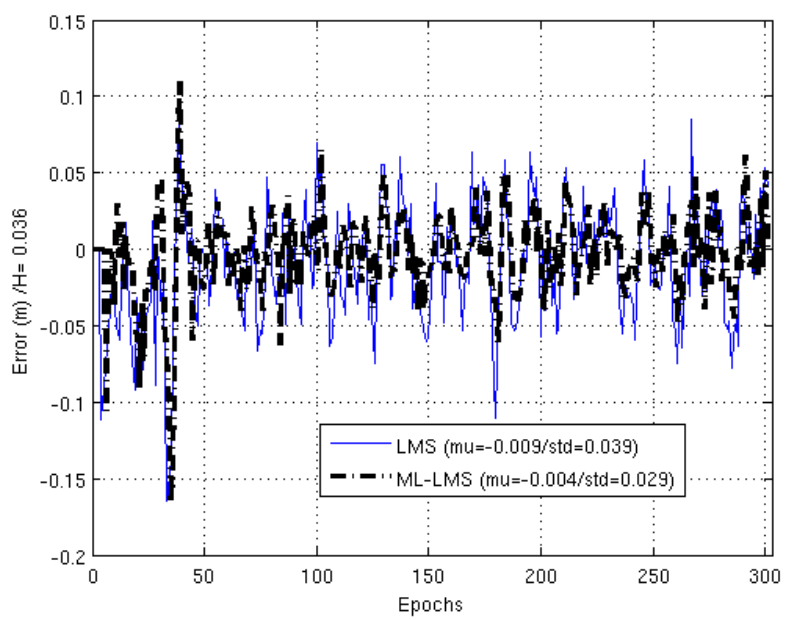

Fig. 1. Convergence time of the three adaptive algorithms when $\mathrm{H}=0.036$

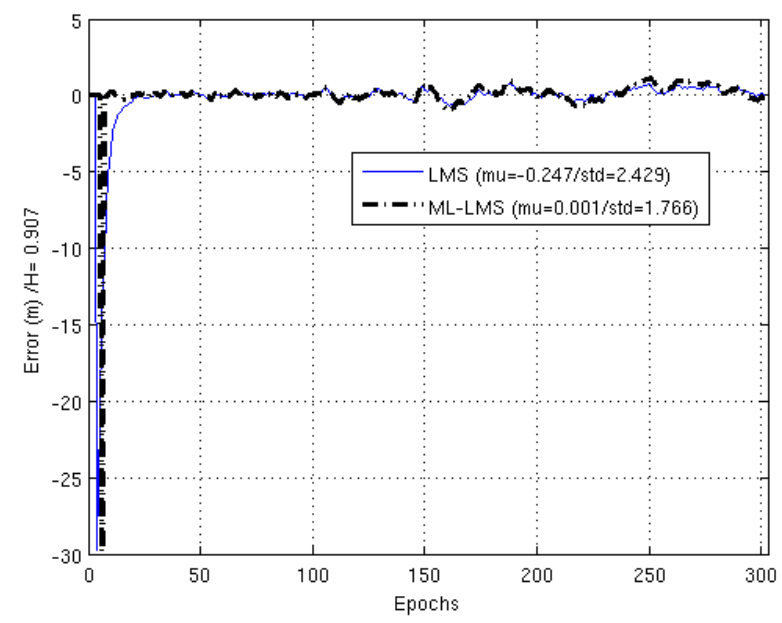

Fig. 2. Convergence time of the three adaptive algorithms when $\mathrm{H}=0.906$

\section{REFERENCES}

[1] A. Papoulis and S. Unnikrishna Pillai, Probability, Random variables, and stochastic processes, 4th Edition, Mc Graw Hill, 2010

[2] https://www.worldscibooks.com/etextbook/p556/p556_chap04.pdf

[4] B. B. Mandelbrot and J. W. Van Ness, "Fractional Brownian Motions, Fractional Noises and Applications," Society for Industrial and Applied Mathematics (SIAM) Review, vol. 10, no. 4, pp. 422-437, Oct. 1968.

[5] R. Scheffer and R. Maciel Filho, "The fractional Brownian motion as a model for an industrial airlift reactor," Chemical Engineering Science, vol. 56, no. 2, pp. 707-711, Jan. 2001.

[6] H. E. Hurst, R. P. Black and Y. M. Sinaika, Long Term-Storage: An Experimental Study, Constable, London, 1965.

[7] M. S. Taqqu, V. Teverovsky and W. Willinger, "Estimators for longrange dependence: an empirical study," Fractals, vol. 3, no. 4, pp. 785798, 1995. Reprinted in C.J.G. Evertsz, H.-O. Peitgen, R.F. Voss (Eds.), Fractal Geometry and Analysis, World Scientific, Singapore, 1996.

[8] C. M. Akujuobi, N. K. Ampah and M. N. O. Sadiku, "An intrusion detection technique based on change in Hurst parameter with application to network security," International Journal of Computer Science and Network Security, vol.7, no.5, pp. 55-64, May 2007.

[9] S. Haykin, Adaptive Filter Theory, 4th edition, Prentice Hall Upper Saddle River, New Jersey, 2002.

[10] M. Kamenetsky and B. Widrow, A Variable Leaky LMS Adaptive Algorithm, Record of the Thirty-Eighth Asilomar Conference on Signals, Systems and Computers, 1:125-128, November 2004.

[11] A. Gupta, and S. Joshi, "A novel least mean squares algorithm for tracking a discrete-time $\mathrm{fBm}$ process," Proceedings of the IEEE Indian Conference, pp. 1-6, Sept. 2006.

[12] W. Y. Ochieng, S. Feng, T. Moore, C. Hill and C. Hide, "User level integrity monitoring and quality control for high accuracy positioning using GPS/INS measurements," Journal of Global Positioning Systems, vol. 7, no. 2, pp. 104-114, 2008.

[13] J. R. Wallis and N. C. Matalas, "Small sample properties of H and Kestimators of the Hurst coefficient h," Water Resources Research, vol. 6, no. 6, pp. 1583-1594, Dec. 1970.

[14] J. R. M. Hosking, "Fractional differencing," Biometrika, vol. 68, no. 1, pp. 165-176, Apr. 1981.

[15] S. Stoev and M. S. Taqqu, "Wavelet estimation for the Hurst parameter in stable processes," Lecture Notes in Physics, vol. 621, pp. 61-87, 2003.

[16] S. M. Kay, Fundamentals of Statistical Signal Processing: Estimation Theory, PTR Prentice Hall, Englewood Cliffs, New Jersey, 1993.

[17] K. Borre and G. Strang, Linear Algebra, Geodesy, and GPS, Wellesley College Publisher, 1997.

[18] J.-P. Montillet, K. Yu, I. Oppermann, "Location performance enhancement with recursive processing of time-of-arrival measurements, Proceedings of IEEE International Symposium on Personal, Indoor and Mobile Radio Communications (PIMRC), pp. 1-5, Sept. 2007.

[19] U. Weinbach , N. Raziq and P. Collier, "Mitigation of periodic GPS multipath errors using a normalised least mean square adaptive filter," Journal of Spatial Science, vol. 54, no. 1, pp. 1-13, 2009.

[20] T. Moore, C. Hill, C. Hide, W. Ochieng, S. Feng, E. Aguado, R. Ioannides, P. Cross and L. Lau, "End-to-end testing of an integrated centimetric positioning test-bed," Proceedings of the ION GNSS, Fort Worth, Texas, Sept. 2007. 